positive impact a short course can have in promoting engagement in Psychiatry by students.

Method. An anonymous questionnaire was distributed amongst medical students, from years 1 to 5 , in the College of Medicine, Alfaisal University, Riyadh, Saudi Arabia. Factors assessed in the survey included demographics, specialty ranking, acceptance ratios, role models and personal experiences, among others. Those who didn't express interest were asked about the lack of exposure to Psychiatry during medical school along with other influential factors that have been studied elsewhere, including those that we hypothesise to be of significance in our study population.

Result. A total of 153 students responded. Positive views towards Psychiatry increased linearly by year (50\% in Year 1 to $90 \%$ in Year 5). 33\% of students selected psychiatry as a top 3 choice with the most significant factors being a unique patient-doctor relationship $(\mathrm{P}<0.05)$, and the challenges faced in the specialty (95\%).

Of the $67 \%$ of students who did not prefer Psychiatry, insufficient exposure to ward experiences and the specialty as a whole were unanimously agreed upon factors. Other deterring factors included lack of instant gratification when treating a psychiatric patient $(72 \%)$, and an underestimation by the non-medical community of a Psychiatrists role (26\%).

Conclusion. Our findings give an optimistic view towards the future of Psychiatry in the region, given the large number of students $(33 \%)$ who consider it in their top 3 choices for a career. However, a larger number of students continue to have a negative view towards Psychiatry, especially due to the lack of exposure to the specialty during medical school. The factors identified in our study should be tackled by medical schools or curriculum provision authorities, as this has shown to be of benefit in studies in other parts of the world.

The impact of psychiatry school on attitudes towards psychiatry in medical students and junior doctors in Pakistan

Raja Adnan Ahmed ${ }^{1, *}$, Sanaa Moledina ${ }^{2}$ and Usama Asad ${ }^{3}$

${ }^{1}$ Cwm Taf Morgannwg University Health Board; ${ }^{2}$ Northamptonshire Healthcare NHS Foundation Trust and ${ }^{3}$ Shaheed Mohtarma Benazir Bhutto Institute of Trauma

${ }^{\star}$ Corresponding author.

doi: 10.1192/bjo.2021.71

Aims. To assess the impact of Psychiatry School on the attitudes towards psychiatry in Pakistani medical students (MS) and junior doctors (JD).

Method. Inspired by the Royal College of Psychiatrists' 'Choose Psychiatry' campaign, an online event by the name of 'Psychiatry Autumn School Pakistan' was held on the 1st of November 2020. The event was promoted through social media and medical students and junior doctors from across Pakistan were invited to attend. Moreover, a panel of British and Pakistani psychiatrists belonging to different sub-specialties was invited to deliver talks. The attendees were provided an insight into psychiatry as a viable career option and were introduced to the training pathways, research opportunities, and the various sub-specialties present within the field.

Participants were requested to complete the 'Attitudes Towards Psychiatry' (ATP-30) questionnaires before and immediately after the event. Individual scores on the questionnaire can range from 30 to 150 and a high score indicates a positive attitude. Statistical analysis was performed using a paired t-test.
Result. 41 attendees (MS $=30$, JD = 11) completed the pre-and post-school survey. The respondents were majorly female (76\%) and from public sector universities (76\%), with an average age of 23 years. The mean ATP score before the course was 119 $(\mathrm{MS}=117, \mathrm{JD}=121)$ which increased by 9 points to $128(\mathrm{MS}=$ $126, \mathrm{JD}=131$ ) after the event. When the two samples were compared using a paired t-test, the difference was statistically significant $\mathrm{p}<0.005$.

Conclusion. We conclude that a psychiatry school can positively influence attitudes towards psychiatry in medical students and junior doctors and our findings are consistent with similar studies done in other countries.

In Pakistan, unfortunately, only $2-4 \%$ of undergraduate students opt for a career in psychiatry owing to insufficient knowledge and awareness about the available treatment modalities and advancement in the field. Hence, such an intervention can greatly enhance recruitment within the profession as it makes psychiatry more accessible and visible as a career choice, generates awareness about the effectiveness and evolution of psychotherapeutic practices, and eradicates misconceptions about the field that prevail among young doctors.

This was the first psychiatry school held in Pakistan and the findings of the study as well the feedback received from the participants and the speakers motivate us to continue campaigning for 'Choose Psychiatry.'

\section{Prevalence and correlates of depression and quality of life among primary caregivers of patients with schizophrenia attending a Nigerian Tertiary Hospital}

Akinloye Akinfala ${ }^{1 \star}$, Oladipo Sowunmi ${ }^{2}$ and Imam Sakeeb ${ }^{2}$

${ }^{1}$ Lancashire \& South Cumbria NHS Foundation Trust and

${ }^{2}$ Neuropsychiatric Hospital Aro

${ }^{\star}$ Corresponding author.

doi: 10.1192/bjo.2021.72

Aims. To determine the prevalence and correlates of depression and quality of life and their relationship among primary caregivers of patients with schizophrenia in a psychiatry specialist hospital.

Method. A total of 138 caregivers of patients diagnosed with schizophrenia attending the outpatient clinic of the Neuropsychiatric Hospital Aro, Abeokuta were recruited. Sociodemographic questionnaire, Mini International Neuropsychiatric Interview (MINIPLUS) (depressive module) and World Health Organization Quality of Life-Bref (WHOQOL-Bref) were administered on the caregivers while Brief Psychiatric Rating Scale (BPRS) was used to measure symptoms severity in the patients.

Result. The mean $( \pm \mathrm{SD})$ age of respondents was 48.3 years $( \pm 14.7), 53.6 \%$ were females and $33.3 \%$ were without partners. The prevalence of depression among the caregivers who participated in the study was $13.8 \%$. Female gender $(\chi 2=5.68, \mathrm{df}=1$, $\mathrm{p}=0.02)$, hailing from a minority tribe $(\chi 2=9.78 \mathrm{df}=1$, $\mathrm{p}<0.01)$, and Previous treatment for mental illness $(\chi 2=8.24$ $\mathrm{df}=1, \quad \mathrm{p}<0.01)$ were associated with depression. Female gender $(\beta=1.35, \mathrm{OR}=3.86, \mathrm{p}=0.03)$, minority tribe $(\beta=1.95$, $\mathrm{OR}=7.03, \mathrm{p}<0.01)$, and previous treatment for mental illness $(B=3.19, \mathrm{OR}=24.21, \mathrm{p}=0.01)$ were independently predictive of depression in the caregivers.

Independent predictors of lower quality of life (QOL) were: Parents/siblings relationship for social relationship domain $(\beta=-7.076, p=0.037)$ and spending more than 35 hours per week for Environmental domain $(\beta=-5.622, p=0.028)$. 